\title{
Szenario-Analyse trifft agentenbasierte Marktsimulation
}

\author{
Entscheidungsunterstützung bei der strategischen Technologieplanung
}

\author{
Christian Stummer, Universität Bielefeld, Fakultät für Wirtschaftswissenschaften, Universitätsstr. 25, 33615 Bielefeld \\ (christian.stummer@uni-bielefeld.de), (1) orcid.org/0000-0001-5495-5740 \\ Markus Günther, Universität Bielefeld, Fakultät für Wirtschaftswissenschaften (markus.guenther@uni-bielefeld.de), (D) orcid.org/0000-0003-1853-3123
}

Während eine Szenario-Analyse die Vorausschau auf alternative Zukünfte erlaubt, bietet eine agentenbasierte Marktsimulation Einblick in das komplexe Verhalten von Märkten. Eine Kombination dieser beiden Methoden kann die Entscheidungsgrundlage für die strategische Technologieplanung verbessern. Dazu werden die Auswirkungen von unterschiedlichen Technologieportfolios auf den Erfolg in zukünftigen Märkten simuliert, wobei die Marktcharakteristika aus den Szenario-Beschreibungen abgeleitet werden und sich die Produkteigenschaften durch die Technologien aus den untersuchten Portfolios ergeben. Zugleich lässt sich auch die Effektivität diverser Strategien zur Markteinführung der neuen Produkte testen. Dieser Beitrag beschreibt einen solchen hybriden Ansatz zur Entscheidungsunterstützung bei der strategischen Technologieplanung und illustriert seine Anwendbarkeit bzw. den praktischen Nutzen anhand eines Beispiels.

\section{Scenario Analysis Meets Agent-Based Market Simulation \\ Decision Support for Strategic Technology Planning}

While scenario analysis allows forecasts of alternative futures, agentbased market simulation provides an insight into the complex behavior of markets. Combining these two methods bears the potential to strengthen the basis for decision making in strategic technology planning. This is achieved by simulating the impact of various technology portfolios on success in future markets, for which market characteristics are derived from the scenarios descriptions and the product attributes are determined through the technologies available in the portfolio under investigation. Moreover, it is also possible to test the effectivity of different strategies for introducing the new products into the market. This paper describes such a multi-method decision support approach for strategic technology planning and illustrates its applicability as well as its practical value with an example.

KEYWORDS: technology planning, agent-based market simulation, scenario analysis

This is an article distributed under the terms of the Creative Commons Attribution License CCBY 4.0 (https://creativecommons.org/licenses/by/4.0/)

https://doi.org/10.14512/tatup.26.3.17

Submitted: 06. 07.2017. Peer reviewed. Accepted: 15. 09.2017

\section{Szenario-Analyse in der Technologie- planung}

Eine Szenario-Analyse schafft „Bilder der Zukunft“, die einem Unternehmen dabei helfen können, sich auf Eventualitäten vorzubereiten (Amer et al. 2013). Sie hat sich vor allem auch in der strategischen Technologieplanung etabliert (Rigby und Bilodeau 2007). Dabei erhebt die Szenario-Analyse nicht den Anspruch, die Zukunft vorherzusagen (Schoemaker 1991), kann aber wertvolle Unterstützung beim Überwinden von Denkgrenzen bieten (Amer et al. 2013). Einen Überblick zur Entwicklung und zu Anwendungsfeldern der Szenario-Analyse geben Van Notten et al. (2003) und Bradfield et al. (2005).

Szenarien können in vielfältiger Weise ausgestaltet sein und dementsprechend auch für unterschiedliche Abstraktionsebenen - beispielsweise als Markt-, Unternehmensumfeld- oder als unternehmensspezifische Szenarien - erstellt werden. In dieser Arbeit folgen wir dem Ansatz von Gausemeier et al. (1998), dessen Nutzen für die strategische Technologieplanung mehrfach in der betrieblichen Praxis demonstriert wurde. Der letzte Prozessschritt in diesem Vorgehensmodell sieht eine Gegenüberstellung der entwickelten Szenarien mit den zur Auswahl stehenden Handlungsoptionen vor. Letztere können zu „Maßnahmenportfolios“ zusammengefasst werden und enthalten beispielsweise Forschungs- und Entwicklungsvorhaben (um etwa die benötigten Technologien zu entwickeln und damit die Funktionalität der Produkte entsprechend zu erweitern), Marketingstrategien (etwa bezüglich der Preissetzung) oder neue Geschäftsmodelle. Die Entscheidungsträger sollen damit bei der Identifikation jenes Maßnahmenportfolios unterstützt werden, das eingedenk der möglichen alternativen „Zukünfte“ am geeignetsten ist.

Gausemeier et al. (2014) nutzen hierfür eine Matrix, wobei jedes Szenario eine eigene Spalte und jedes Maßnahmenportfolio eine eigene Zeile zugewiesen bekommt. Die Matrixeinträge reichen von Doppel-Plus für „,sehr hohe Konsistenz“ bis Doppel-Minus für ,sehr hohe Inkonsistenz“ und spiegeln holistisch die (vermutete) Eignung eines Maßnahmenportfolios für 


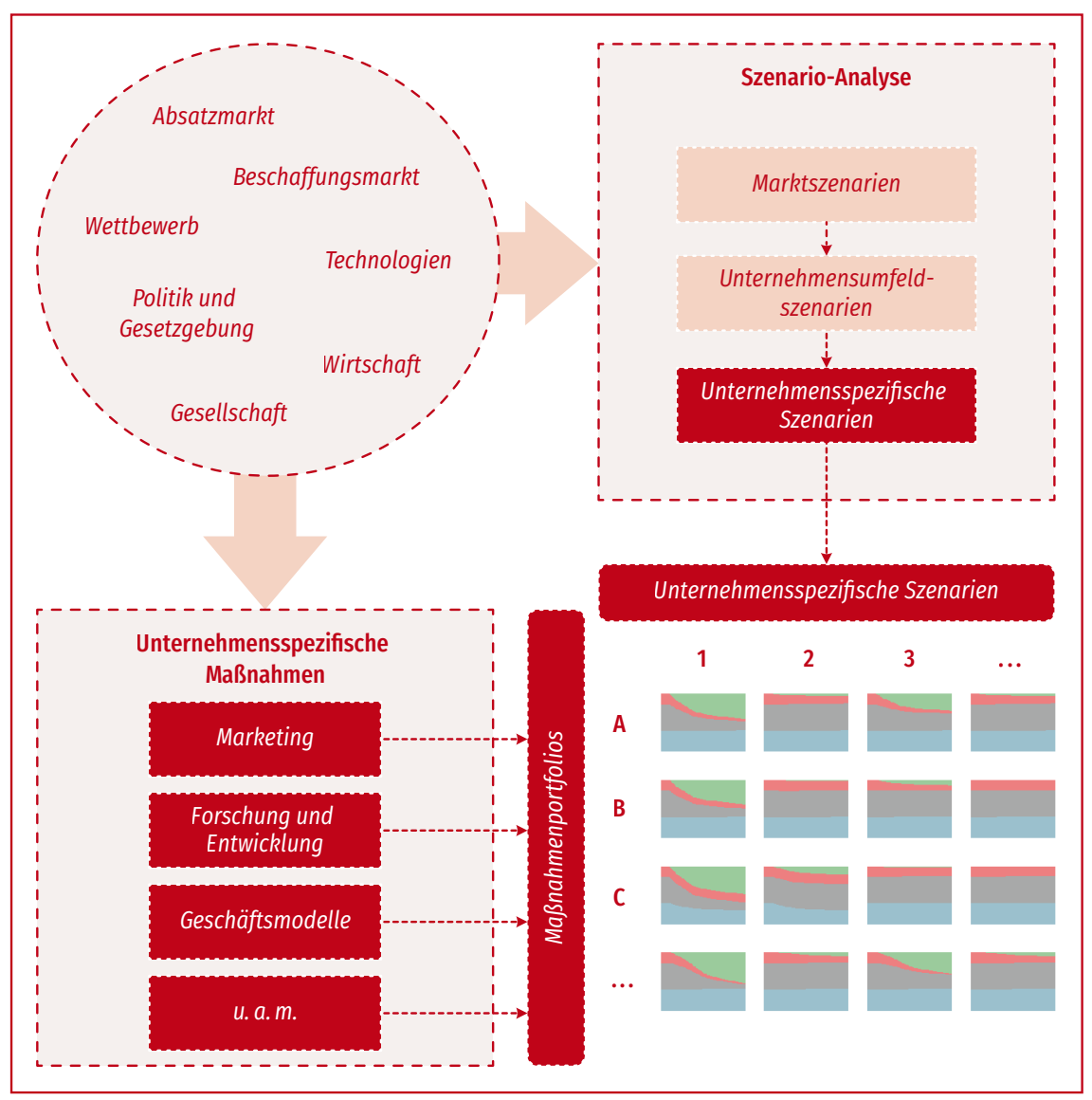

Abb.1: Planungsunterstützung durch Kombination aus Szenario-Analyse und Marktsimulation.

Quelle: Eigene Darstellung

jede Kombination aus Szenario und Maßnahmenportfolio fundierte Informationen über die voraussichtliche Marktentwicklung im Zeitablauf (in diesem Beispiel die Entwicklung der Marktanteile) erhalten; vgl. den rechten unteren Quadranten in Abbildung 1.

Dazu werden reale Prozesse, Ereignisse und (Wirkungs-)Systeme in einer Modellwelt nachgebildet, sodass auf diesen virtuellen Märkten Simulationsexperimente (z. B. zur Umsetzung einzelner Maßnahmenportfolios) durchgeführt werden können (Harrison et al. 2007). Angesichts der Komplexität bzw. der Neuheit der Märkte sind weder analytische noch empirische Ansätze als Alternative zur vorgeschlagenen Computer-Simulation anwendbar. Ob jedoch ein System-Dynamics-Ansatz oder eine agentenbasierte Simulation geeigneter erscheint - eine Gegenüberstellung der beiden Ansätze bieten etwa Borshchev und Filippov (2004) -, wurde anhand der Richtlinien von Rand und Rust (2011) evaluiert. Demnach eignet sich eine agentenbasierte Simulation, wenn (i) eine mittlere Anzahl von Akteuren als Agenten (z. B. Konsumenten) abgebildet werden soll, (ii) lokale Interaktionen (z. B. die Informationsweitergabe durch Mundpropaganda) eine Rolle spielen, (iii) die Agenten heterogene Eigen-

ein Szenario wider. Dieser Prozessschritt ist Ausgangspunkt für den, im weiteren Verlauf dieses Aufsatzes eingeführten, hybriden (Multimethoden-)Ansatz.

\section{Erweiterung um eine Markt- simulation}

Unsere Erweiterung der Szenario-Analyse sieht vor, die derzeitige Praxis der auf recht groben bzw. individuellen Einschätzungen beruhenden Bewertung der Passung zwischen den Szenarien und den Maßnahmenportfolios durch die deutlich reichhaltigeren Ergebnisse einer Marktsimulation zu ersetzen. Die Simulation erlaubt dabei die unmittelbare Berücksichtigung konkreter Markteigenschaften (aus denen sich unterschiedliche Markterschließungsmaßnahmen ergeben können), sie ermöglicht eine Betrachtung der Marktdurchsetzung neuer Produkte (mit Produkteigenschaften, die auf Technologien aus dem festzulegenden Technologieportfolio basieren) und sie eröffnet vielfältige Analysemöglichkeiten. Abbildung 1 illustriert das Gesamtkonzept. Der besondere Mehrwert der Erweiterung um eine Marktsimulation liegt somit darin, dass die Entscheidungsträger für schaften (z. B. bezüglich ihrer Präferenzen) haben, (iv) sich aus der Modellumwelt, in der sich die Agenten bewegen, Implikationen auf das Verhalten ergeben (z. B. räumliche Distanzen), (v) der Zeitablauf von Relevanz ist und (v) sich (manche) Agenten im Simulationsablauf adaptiv (etwa als Reaktion auf Veränderungen in ihrem Umfeld) verhalten.

Alle diese Voraussetzungen sind in unserer Anwendung gegeben, weshalb wir die Szenario-Analyse mit einer agentenbasierten Marktsimulation erweitert haben.

Während agentenbasierte Simulationen zur Untersuchung von Diffusionsverläufen in der Literatur mittlerweile recht populär geworden sind (eine Übersicht bieten u. a. Kiesling et al. 2012 oder Wakolbinger et al. 2013), haben sie sich in der Praxis erst in geringem Maße durchgesetzt. Erklärbar ist das teils durch die Herausforderungen, die sich durch die passende Parametrisierung von agentenbasierten Simulationen für reale Märkte ergibt (ein Anwendungsbeispiel, in dem dies gelungen ist, wird von Stummer et al. 2015 a beschrieben). Der in diesem Beitrag vorgestellte hybride Ansatz trägt zu diesem Forschungsfeld bei, indem die Kombination mit einer Szenario-Analyse zum Zwecke der strategischen Technologieplanung vorgeschlagen wird. 


\section{Elemente der agenten- basierten Marktsimulation}

In einer solchen agentenbasierten Simulation werden die relevanten Akteure durch Agenten repräsentiert. Dies können in dem von uns betrachteten Kontext Personen (z. B. Konsumenten), Unternehmen (z. B. Produzenten oder Intermediäre bzw. Händler), der Staat, Medien und andere mehr sein. Jeder Agent (und damit insbesondere auch jeder einzelne Konsumenten-Agent) kann individuelle Eigenschaften (z. B. eigene Präferenzen für bestimmte Produkteigenschaften) aufweisen, lokale (private) Informationen (z. B. Einschätzungen über die Ausprägung bestimmter Eigenschaften eines bestimmten Produkts) besitzen und bei Bedarf (z. B. weil das eigene Produkt im Laufe der Simulation gerade defekt geworden ist) selbstständig Entscheidungen treffen

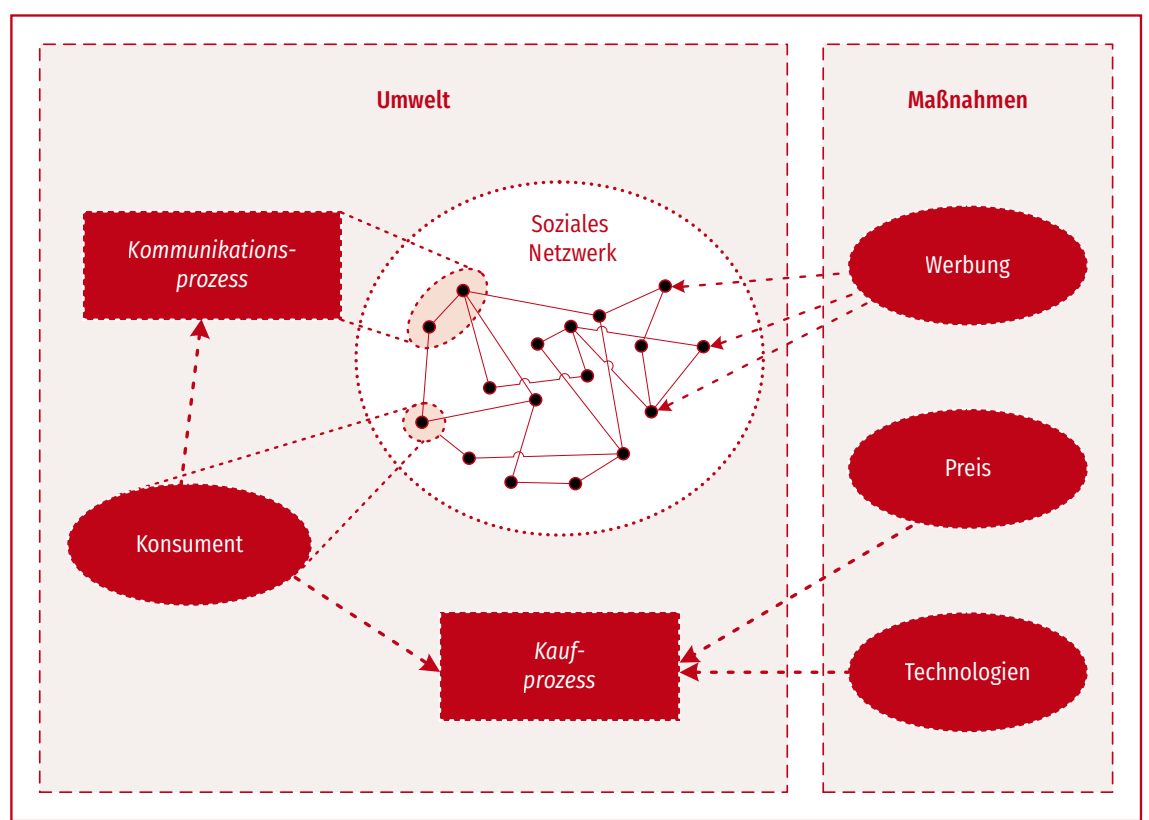

Abb.2: Elemente des agentenbasierten Marktmodells. spiel ein Ersatzprodukt kaufen). Darüber

hinaus können Agenten mit anderen Agenten in Interaktion treten und zwar sowohl mit Agenten desselben Typs (wenn sich beispielsweise zwei Konsumenten-Agenten über ihre Erfahrungen mit einem Produkt austauschen) als auch mit Agenten eines anderen Typs (z. B. ein Konsumenten-Agenten mit einem Händler-Agenten beim Produktkauf).

Die berücksichtigten Typen von Agenten, deren Anzahl, ihre individuellen Eigenschaften und anderes mehr werden durch die Charakteristika des Marktes bzw. die zu untersuchende Fragestellung bestimmt. Dementsprechend gibt es auch nicht das eine (einzige) Modell, das auf jede Anwendung passen würde. Im Folgenden wird beispielhaft ein Marktmodell beschrieben und in Abbildung 2 schematisch dargestellt, das zuvor bereits in ähnlicher Weise im Rahmen des Spitzenclusterprojekts ,it's owl“ (Intelligente Technische Systeme OstWestfalenLippe) eingesetzt worden ist (dieses Vorgängermodell wird von Stummer et al. 2015 b und Günther et al. 2017 beschrieben) und je nach Anwendungsfall angepasst bzw. erweitert werden kann.

Unternehmen bringen neue Produkte auf den Markt, die sich bezüglich ihrer Eigenschaften (z. B. ihrer Funktionalität oder im Preis) von den bisher verfügbaren Angeboten unterscheiden. Die Produkteigenschaften werden wiederum durch die in den Unternehmen vorhandenen Technologien determiniert. Aus Sicht der Unternehmen sind sie damit (auch) Resultat früherer Technologieplanungen, während die Konsumenten im Kaufprozess natürlich vor allem die Produkteigenschaften (z. B. Funktionalität oder Qualität und natürlich den Preis) wahrnehmen und die dabei eingesetzten Technologien per se in der Regel keine entscheidende Rolle spielen.

Konsumenten-Agenten kommt in diesem Marktmodell eine entsprechend zentrale Rolle zu. Sie haben neben einer geografischen Position insbesondere Präferenzen für Produkteigenschaften sowie eine (wenn auch nicht unbedingt korrekte) Einschätzung, wie gut die zur Wahl stehenden Produktalternativen in Bezug auf diese Eigenschaften bzw. Kriterien abschneiden. Während sie die Eigenschaften jener Produkte gut kennen, die sie besitzen oder besessen haben und mit denen sie daher bereits eigene Erfahrungen gemacht haben, basiert ihre Einschätzung der anderen Produkte entweder auf Gesprächen mit anderen Konsumenten-Agenten und/oder auf Informationen aus der Werbung. Wenn neue Produkte mit zusätzlichen Produkteigenschaften in den Markt eingeführt werden, muss den Konsumenten das Vorhandensein dieser Eigenschaften daher erst bewusstgemacht werden, um bei der Auswahlentscheidung berücksichtigt werden zu können.

Mundpropaganda erfolgt in diesem Modell ausschließlich bidirektional zwischen Konsumenten-Agenten, die in einem sozialen Netzwerk direkt miteinander verbunden sind. Dazu haben wir ein skalenfreies Netzwerk nach Barabási und Albert (1999) implementiert und es analog zum Ansatz von Stummer et al. (2015 a) um eine geografische Komponente erweitert, sodass die Wahrscheinlichkeit einer Verbindung zwischen zwei Konsumenten - wie etwa von Latané et al. (1995) beschrieben - mit ihrer geografischen Nähe steigt (eine Diskussion der Vor- und Nachteile von Netzwerktypen findet sich bei Kiesling et al. 2012). Neben der Positionierung im sozialen Netzwerk hat jeder Konsumenten-Agent auch ein individuelles Kommunikationsverhalten, das die Kommunikationshäufigkeit oder die Bewertung der erhaltenen Informationen in Abhängigkeit vom jeweiligen Gesprächspartner bestimmt. 
Wenn der Kauf eines neuen Produkts ansteht, erkundigen sich die Konsumenten-Agenten allenfalls nochmals intensiver in ihrem sozialen Umfeld über die Vor- und Nachteile der dort bekannten Produkte und entscheiden sich dann für einen Vertriebskanal bzw. eine Verkaufsstelle, wobei auch das Vorgehen bei dieser Auswahl - unter Berücksichtigung der geografischen Nähe und/oder bisheriger Erfahrungen und Empfehlungen aus dem eigenen Bekanntenkreis - recht komplex sein kann. Verkaufsstellen haben dazu einen geografischen oder kundengruppenspezifischen Einzugsbereich. Für jedes der zur Wahl stehenden Produkte wird der potenzielle Nutzen anhand einer Nutzenfunktion berechnet, in die neben den eigenen Einschätzungen bezüglich der (bekannten) Produkteigenschaften sowie den je-
Technologien entscheiden, die bestimmte Eigenschaften bzw. Funktionen eines solchen Kühlschranks möglich machen. Diese Entscheidung hängt nicht zuletzt davon ab, ob ein hinreichend großer Markt vorhanden ist, um die Entwicklung (oder Beschaffung) der nötigen Technologien zu rechtfertigen.

Wir betrachten in unserem vereinfachten Anwendungsbeispiel im Folgenden lediglich die zwei in ihrer Ausprägung extremen unternehmensspezifischen Szenarien. Im ersten Szenario stehen die Konsumenten des modellierten Zielmarktes solch einem intelligenten Produkt offen gegenüber, während ihm im zweiten Szenario viele Konsumenten skeptisch bis ablehnend begegnen (etwa auf Grund von Datenschutzbedenken oder Angst vor Kontrollverlust).

\section{Dieser hybride Ansatz eignet sich vor allem für die Technologiefolgen- abschätzung aus Unternehmenssicht.}

weiligen Präferenzen auch der soziale (Gruppen-)Druck einfließen kann. Das Produkt mit dem höchsten subjektiven Nutzen wird schließlich gekauft; mit etwas zeitlicher Verzögerung macht der Konsumenten-Agent dann eigene Erfahrungen mit dem Produkt und passt seine Einschätzung an diese Erfahrungen an. Die dem Kaufentscheidungsprozess zugrundeliegende Nutzenfunktion ist im einfachsten Fall additiv-linear, kann aber auch jede andere praktikable Form annehmen (z. B. die Entscheidungskriterien multiplikativ verknüpfen).

Werbung zielt auf die Beeinflussung des Informationsstands der Konsumenten-Agenten ab bzw. macht auf ein neues Produkt bzw. neue Produkteigenschaften aufmerksam. Für die Massenwerbung, die gegebenenfalls auf eine bestimmte geografische Region oder eine Zielgruppe fokussiert, wird in unserem Modell ein zufällig ausgewählter Teil der adressierten Konsumenten-Agenten mit den Werbe-Informationen konfrontiert. Prozedural entspricht dies einem unidirektionalen Informationsfluss, in dem nur der Werbe-Agent den Konsumenten-Agenten beeinflusst. Des Weiteren können Preise bzw. allenfalls auch adaptive Preisstrategien festgelegt werden.

\section{Anwendungsbeispiel}

Das Anwendungsbeispiel ist aus dem Kontext des Spitzenclusterprojekts „it's owl“ entlehnt, jedoch bezüglich der Parametrisierung maßgeblich verändert. Unterstützt werden soll darin ein Unternehmen, das die Entwicklung bzw. Übernahme neuer Technologien plant, um damit die eigene Angebotspalette um ein vernetztes, intelligentes Produkt (ein Gebrauchsgut im mittleren bis gehobenen Preissegment) zu erweitern. Beispielsweise könnte dies ein ,,intelligenter Kühlschrank “ sein. ${ }^{1}$ Das Unternehmen muss dabei im Zuge der strategischen Technologieplanung unter anderem über die Entwicklung (oder ggf. den Zukauf) von
Angeboten werden Produkte für die Segmente „Discount“, „Standard“ und „Premium“. Die Produkte unterscheiden sich dabei in Bezug auf die Attribute Preis, Funktionalität und Qualität, wobei die Ausprägung der Eigenschaften von Discount zu Premium zunehmen (d.h. Produkte aus dem Discount-Segment haben einen niedrigeren Preis, bieten aber auch geringere Funktionalität und Qualität als Produkte aus dem Standard-Segment, usw.).

Die Konsumenten aus dem Anwendungsbeispiel lassen sich grob in vier Gruppen einteilen. Preisbewusste Konsumenten achten bei ihrer Kaufentscheidung vor allem auf den Preis. Technikaffine Käufer sind dagegen besonders an (neuen) Produkten mit großem Funktionsumfang interessiert. Premium-Käufer werden wiederum vor allem von prestigeträchtigen und meist qualitativ hochwertigen Produkten angesprochen; der Preis spielt für sie eine untergeordnete Rolle bzw. zeigt sich sogar ein leichter Veblen-Effekt (d.h. die Nachfrage erhöht sich mit steigendem Preis). Die meisten Konsumenten zählen schließlich zur „breiten Masse“, die - wenn auch mit unterschiedlichen Gewichtungen - einen ausgewogenen Mix an Produkteigenschaften sucht.

Getestet werden im Folgenden vier Maßnahmenportfolios. In jedem davon wird nach einem Jahr - zusätzlich zu den bestehenden herkömmlichen Produkten - ein intelligentes (,smartes“) Produkt zu einem etwas höheren Preis auf den Markt gebracht.

1 Eine ausführliche Diskussion von Produktintelligenz bzw. den dabei zu unterscheidenden Intelligenzdimensionen würde den Rahmen dieses Beitrags sprengen. Im Weiteren wird für die Zwecke dieses Beispiels unter einem intelligenten Produkt ein Produkt verstanden, in dem Intelligenz generierende Technologien eingesetzt werden, um zusätzliche Funktionen zur Verfügung zu stellen. Im Beispiel mit dem intelligenten Kühlschrank wären das etwa eine Sensorik zur Erfassung des Inhalts, die natürlich-sprachliche Kommunikation mit den Nutzern der Küche, die Kapazität für die Verarbeitung der erhaltenen Informationen sowie die Anbindung an einen Bestell- und Lieferservice für Lebensmittel. 
Das neue intelligente Produkt basiert entweder auf dem Standard- oder auf dem Premium-Produkt. Des Weiteren werden in den Maßnahmenportfolios zwei verschiedenen Marketingstrategien ausprobiert. Die erste, aufwändigere, geht von einem Vorfeldmarketing aus, das kurz vor der Produkteinführung gestartet wird und sich über die Markteinführungsphase hinweg erstreckt. Danach wird für das neue Produkt jeden Monat eine Werbekampagne durchgeführt. In der zweiten, weniger aufwändigen, Marketingstrategie erfolgt nur ersteres; Werbung gibt es somit nur zur Markteinführungsunterstützung. Die Bezeichnung der Maßnahmenportfolios in Abbildung 3 verweist auf die darin enthal-

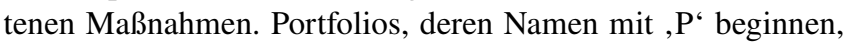
führen ein (neues) intelligentes Produkt mit der Qualität und der Basis-Funktionalität der Premium-Linie ein, , $S$ ‘ steht für das intelligente Produkt der Standard-Linie. Wenn dann sowohl Vorfeldmarketing als auch laufendes Marketing betrieben wird, enthält der Name des Maßnahmenportfolios ein ,++', wenn nur Vorfeldmarketing betrieben wird, ein , ${ }^{\text {' }}$.

In Abbildung 3 werden die Marktanteile der verschiedenen Produkte in den einzelnen Kombinationen von Maßnahmenportfolios und Szenarien über einen Zeithorizont von elf Jahren zusammengefasst. Implementiert wurde die Simulation mit dem Simulationstool AnyLogic 7.0.3.

In den Ergebnissen fällt die geringe Verbreitung der intelligenten Produkte im schwierigen Unternehmensumfeld von Szenario 2 auf. Selbst das intelligente Premium-Produkt verbreitet sich nur sehr verhalten, wobei vermehrtes Marketing immerhin zu einem etwas höheren Markterfolg führt. Das entsprechende Produkt der Standard-Linie setzt sich dagegen überhaupt nicht durch.

Deutlich erfolgreicher sind intelligente Produkte im Szenario 1, wenngleich sich auch hier zeigt, dass dafür intensive Marketingbemühungen nötig sind. Wenn das Portfolio insgesamt stimmig gewählt ist (wie in Maßnahmenportfolio $\mathrm{P}++$ ), dann kann trotz des höchsten Preises eine kritische Masse unter den Konsumenten erreicht werden, sodass letztlich - wegen der Netzwerkeffekte am Markt - zusätzlich zu den meisten Premium-Käufern auch viele vormalige Standard-Käufer gewonnen werden. Das Maßnahmenportfolio S++ führt demgegenüber dazu, dass Premium-Käufer zum Standard-Produkt wechseln. Da im Premium-Kundensegment aber besonders hohe Deckungsbeiträge gemessen als Differenz zwischen Erlös und den variablen Kosten - erzielt werden, ist dies eigentlich unerwünscht.

In diesem Anwendungsbeispiel ist es somit empfehlenswert, Intelligenz generierende Technologien (zunächst) für das Premium-Segment vorzusehen und die intelligenten Produkte mit Werbeaktivitäten nicht nur während der Markteinführung, sondern auch danach noch über einen längeren Zeitraum zu unterstützen. Der entscheidende Informationsgewinn aus dieser Marktsimulation ist aber nicht nur die naheliegende Erkenntnis, dass sich ein solcher Effekt einstellen kann, sondern insbesondere wie groß dieser Effekt für bestimmte alternative Marketingmaßnahmen ausfällt. Dies ließe sich nun durch weitere Simulationen für andere, an Portfolio $\mathrm{P}++$ angelehnte Maßnahmenportfolios untersuchen bzw. tiefergehend analysieren.

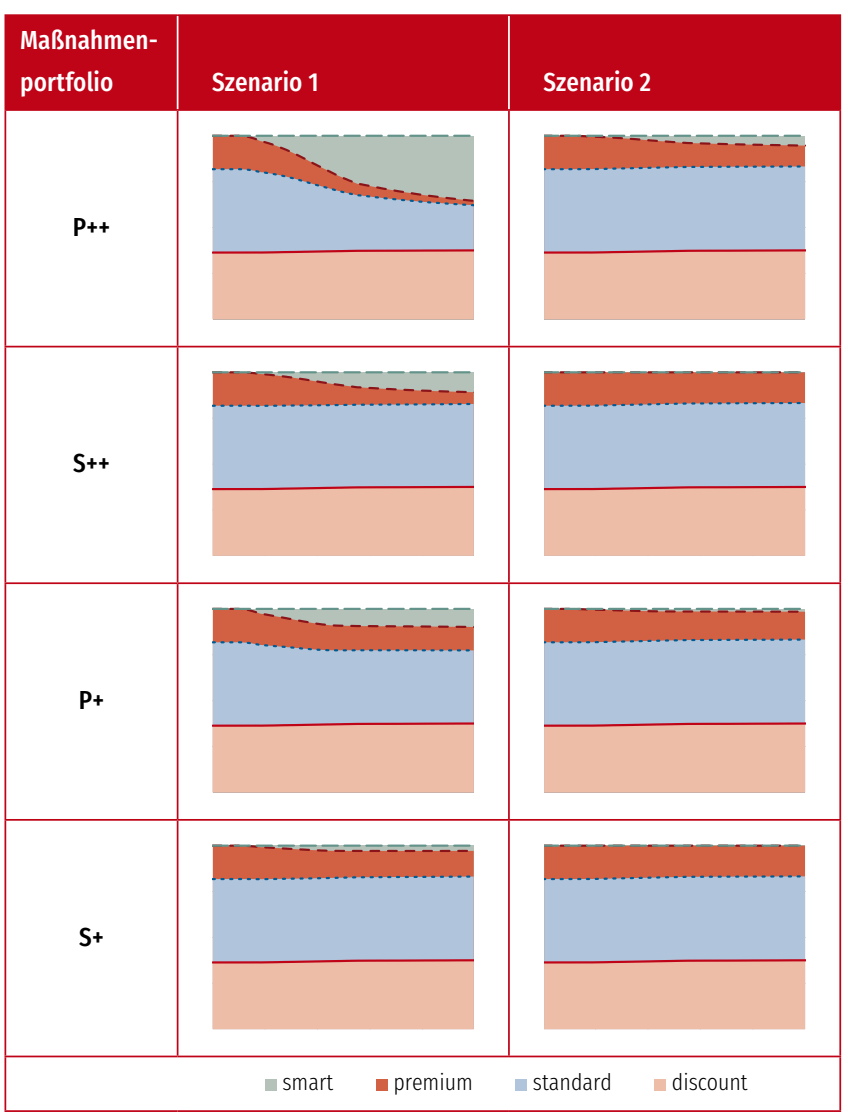

Abb.3: Marktanteilsverläufe für Produkte aus Anwendungsbeispiel.

Quelle: Eigene Darstellung

\section{Fazit}

Die in diesem Beitrag vorgestellte Kombination einer klassischen Szenario-Analyse zur Beschreibung der Charakteristika zukünftiger Märkte mit einer darauf aufsetzenden agentenbasierten Marktsimulation bietet eine fundierte Entscheidungsgrundlage für die strategische Technologieplanung. Dieser hybride Ansatz eignet sich dabei vor allem für die Technologiefolgenabschätzung aus Unternehmenssicht. Manager bekommen damit einerseits ein Instrument an die Hand, das es erlaubt, Strategien zur „Eroberung“ bestehender oder neuer Märkte vorab zu evaluieren und diese Informationen in die Auswahl eines Technologieportfolios einfließen zu lassen. Durch die Simulation für mehrere Szenarien mit unterschiedlichen Marktentwicklungen bekommen Manager andererseits aber auch die Chance, auf die Robustheit ihrer Entscheidungen in alternativen Zukünften zu achten bzw. werden sie dafür sensibilisiert. Darüber hinaus wird die Markteinführung neuer Produkte nicht isoliert, sondern eingebettet in ein komplexes (Markt-)System mit Mitbewerbern und gegebenenfalls auch Intermediären bzw. Vertriebspartnern untersucht, was die Analyse der Auswirkungen der eigenen Technologieentscheidungen bzw. der sich daraus ergebenden zukünftigen Marktangebote auf die anderen Marktteilneh- 
mer bzw. die Marktentwicklung erlaubt. Schließlich hat der hier vorgestellte Ansatz Potenzial für die Untersuchung einer Reihe weiterer, insbesondere betriebswirtschaftlicher Fragestellungen, wie zum Beispiel in Bezug auf allenfalls notwendig werdende Änderungen in der Lieferkette oder den Vertriebskanälen, die Nützlichkeit von strategischen Allianzen etc.

Der Einsatz einer agentenbasierten Marktsimulation bringt andererseits auch Herausforderungen mit sich. Die größte davon ist sicherlich die passende Parametrisierung der Simulation für den jeweiligen Anwendungsfall. Empfehlenswert ist zudem eine Sensitivitätsanalyse der zentralen Parameter. Sollte sich zeigen, dass die Simulationsergebnisse nicht hinreichend robust sind, so ist ganz besonderes Augenmerk auf die dafür verantwortlichen, kritischen Parameter zu legen und die Anwender sind über die damit verbundene Ungewissheit zu informieren.

\section{Literatur}

Amer, Muhammad; Daim, Tugrul U.; Jetter, Antonie (2013): A Review of Scenario Planning. In: Futures 46, S. 23-40. DOI: 10.1016/j.futures.2012.10.003.

Barabási, Albert-László; Albert, Réka (1999): Emergence of Scaling in Random Networks. In: Science 286 (5439), S. 509-512. DOI: 10.1126/science. 286.5439.509.

Borshchev, Andrei; Filippov, Alexei (2004): From System Dynamics and Discrete Event to Practical Agent Based Modeling: Reasons, Techniques, Tools.

In: Michael Kennedy, Graham W. Winch, Robin S. Langer, Jennifer I. Rowe und Joan M. Yanni (Hg.): Proceedings of the 22nd International Conference of the System Dynamics Society. Oxford, UK: System Dynamics Society, $23 \mathrm{~S}$.

Bradfield, Ron; Wright, George; Burt, George; Cairns, George; Van Der Heijden, Keese (2005): The Origins and Evolution of Scenario Techniques in Long Range Business Planning. In: Futures 37 (8), S. 795-812. D0I: 10.1016/j. futures.2005.01.003.

Gausemeier, Jürgen; Fink, Alexander; Schlake, Oliver (1998): Scenario Management: An Approach to Develop Future Potentials. In: Technological Forecasting \& Social Change 59 (2), S. 111-130. DOI: 10.1016/S00401625(97)00166-2.

Gausemeier, Jürgen; Amshoff, Benjamin; Dülme, Christian; Kage, Martin (2014): Strategische Planung von Marktleistungen im Kontext Industrie 4.0. In: Jürgen Gausemeier (Hg.): Vorausschau und Technologieplanung. Tagungsband vom 10. Symposium für Vorausschau und Technologieplanung (SVT). Paderborn: HNI-Verlagsschriftenreihe, S. 5-36.

Günther, Markus; Lüpke, Lars; Stummer, Christian (2017): Cross-over between Scenario Analysis and Agent-Based Market Simulation for Technology Planning. In: Proceedings of PICMET '17: Technology Management for Interconnected World. Portland: International Conference on Management of Engineering and Technology (PICMET), S. 1-9.

Harrison, J. Richard; Zhiang, Lin; Carroll, Glenn R.; Carley, Kathleen M. (2007): Simulation Modelling in Organizational and Management Research. In: Academy of Management Review 32 (4), S. 1229-1245. DOI: 10.5465/ AMR.2007.26586096.

Kiesling, Elmar; Günther, Markus; Stummer, Christian; Wakolbinger, Lea M. (2012) Agent-Based Simulation of Innovation Diffusion: A Review. In: Central European Journal of Operations Research 20 (2), S. 183-230. DOI: 10.1007/ s10100-011-0210-y.
Latané, Bibb; Liu, James H.; Nowak, Andrzej; Bonevento, Michael; Zheng, Long (1995): Distance Matters: Physical Space and Social Impact. In: Personality and Social Psychology Bulletin 21 (8), S.795-805. DOI: 10.1177/0146167295218002.

Rand, William; Rust, Roland T. (2011): Agent-Based Modeling in Marketing: Guidelines for Rigor. In: International Journal of Research in Marketing 28 (3), S. 181-193. DOI: 10.1016/j.ijresmar.2011.04.002.

Rigby, Darrell; Bilodeau, Barbara (2007): A Growing Focus on Preparedness. In: Harvard Business Review 85 (7-8), S. 21-22.

Schoemaker, Paul J. H. (1991): When and How to Use Scenario Planning: A Heuristic Approach. In: Journal of Forecasting 10 (6), S.549-564. DOI: 10.1002/for.3980100602

Stummer, Christian; Kiesling, Elmar; Günther, Markus; Vetschera, Rudolf (2015 a): Innovation Diffusion of Repeat Purchase Products in a Competitive Market: An Agent-Based Simulation Approach. In: European Journal of Operational Research 245 (1), S. 157-167. D0I: 10.1016/j.ejor.2015.03.008.

Stummer, Christian; Lüpke, Lars; Günther, Markus (2015 b): Intelligente Produkte, Zukunftsszenarien und Agenten. Eine Marktsimulation zur Entscheidungsunterstützung bei der strategischen Technologieplanung. In: Jürgen Gausemeier (Hg.): Vorausschau und Technologieplanung, Tagungsband vom 11. Symposium für Vorausschau und Technologieplanung (SVT). Paderborn: HNI-Verlagsschriftenreihe, S. 449-470.

Van Notten, Philip W. F.; Rotmans, Jan; Van Asselt, Marjolein B. A.; Rothman, Dale S. (2003): An Updated Scenario Typology. In: Futures 35 (5), S. 423-443. DOI: 10.1016/S0016-3287(02)00090-3.

Wakolbinger, Lea M.; Stummer, Christian; Günther, Markus (2013): Market Introduction and Diffusion of New Products: Recent Developments in AgentBased Modeling. In: International Journal of Innovation and Technology Management 10 (5), 1340015 (19 S.). DOI: 10.1142/S0219877013400154.

\section{Forschungsdaten}

Die Forschungsdaten wurden im Rahmen einer Kooperation von einem Unternehmen zur Verfügung gestellt. Die Parameter für das Anwendungsbeispiel in dieser Arbeit sind an diese Daten angelehnt, wurden aber maßgeblich verändert.

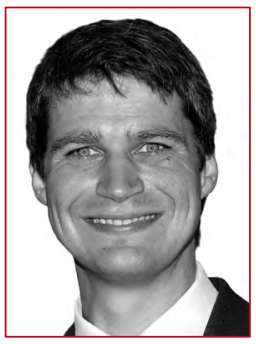

\section{PROF.DR. CHRISTIAN STUMMER}

beschäftigt sich u. a. mit Fragen der Markteinführung bzw. -durchsetzung von Innovationen. Er arbeitet mit analytischen Methoden und setzt dabei häufig auch agentenbasierte Marktsimulationen ein. Derzeit interessieren ihn vor allem ökonomische Implikationen durch die zunehmende Vernetzung und Intelligenz von Produkten.

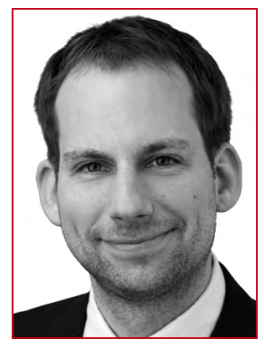

\section{J.-PROF. DR. MARKUS GÜNTHER}

hat aktuell die Vertretung der Professur für Innovations- und Technologiemanagement an der Universität Bielefeld inne und beschäftigt sich $u$. a. mit der Diffusion von Innovationen und Technologien insbesondere mittels agentenbasierter simulation. 